\title{
Pediatricians' Knowledge and Awareness on Pre-surgical Orthopedics in Newborns with Cleft Lip and Palate
}

\author{
(D) Kevser Kurt Demirsoy’1, (1) Süleyman Kutalmış Büyük2, (D) Tuğçe Alpaydın² \\ ${ }^{1}$ Nevşehir Hacı Bektaş Veli University Faculty of Dentistry, Department of Orthodontics, Nevşehir, Turkey \\ 2Ordu University Faculty of Dentistry, Department of Orthodontics, Ordu, Turkey
}

\begin{abstract}
Aim: The aim of this study is to determine the level of knowledge and awareness of pediatricians about pre-surgical orthopedic treatment and nasoalveolar molding (NAM) of newborns with cleft lip and/or palate (CL/P).

Materials and Methods: We conducted a 10-item questionnaire and sent emails to 600 pediatricians in Türkiye. The title, professional experience, institution of employment of the participants and their knowledge level about preoperative maxillary orthopedic treatment and NAM practices of newborns with $\mathrm{CL} / \mathrm{P}$ were evaluated.

Results: One hundred-twelve pediatricians responded to the questionnaire. There was no statistically significant difference in the knowledge level regarding the preoperative treatment of newborns with $C L / P$ between experienced and inexperienced pediatricians ( $p>0.05)$. When the knowledge levels about preoperative treatment and NAM applications of newborns with CL/P were evaluated, no statistically significant difference was found between pediatricians with more or less than 5 years' experience $(p>0.05)$. All pediatricians who participated in the questionnaire reported that they referred newborns with $\mathrm{CL} / \mathrm{P}$ to at least one specialist (orthodontist, dentist or plastic surgeons). The most frequently referred specialist group was plastic surgeons (84\%), followed by orthodontists (52\%) and dentists (39\%). A linear regression was found between the number of cases seen and the professional experience year of pediatricians.

Conclusion: Increasing the awareness and knowledge of pediatricians about NAM, pre-surgical orthopedic and other treatment alternatives in newborns with $\mathrm{CL} / \mathrm{P}$ is very important for the success of $\mathrm{CL} / \mathrm{P}$ treatment. Newborns with $\mathrm{CL} / \mathrm{P}$ should be referred to an orthodontist by pediatricians as soon as possible after birth to initiate the treatment process.
\end{abstract}

Keywords: Cleft lip and palate, nasoalveolar molding, pediatrician, newborn

\section{Introduction}

Non-syndromic cleft lip/palate $(\mathrm{CL} / \mathrm{P})$ is one of the most common congenital craniofacial abnormalities worldwide. Non-syndromic CL/P's etiology is multifactorial based on the interaction of genetic and environmental factors (1). $\mathrm{CL} / \mathrm{P}$ may be associated with one of many syndromes, as approximately 275 syndromes with orofacial clefts as a primary feature have been identified. Etiologies include mutation of a single genetic locus, teratogenic factors, and chromosomal abnormalities (2). The development of facial structures occurs between the fourth and twelfth weeks of pregnancy, with the left and right sides of the facial structures fusing in the middle of the fetus' body during this time; however, if the parts of the face do not fuse properly due genetic or environmental factors, craniofacial clefts will occur (3-5). 
CL/P should be treated with a multidisciplinary approach involving many specialists including pediatricians, obstetrician-gynecologists, speech therapists, plastic surgeons, orthodontists, and dentists. The first and most important obstacle in the treatment of infants born with CL/P is nutrition. As a general method, this problem is addressed with passive feeding plates. It is also possible to approximate the cleft segments closer to each other and to extend the columella by making active regulatory additions to the feeding plates. This is the best method to improve facial aesthetics to date (3). The treatment approach of the clinical team following the patient will determine the individual treatment protocol. Although newborns with CL/P undergo many reconstructive surgeries throughout their lives, they may continue to have aesthetic challenges as they grow up. Therefore, determining the right treatment approach is one of the most important missions of the clinical team. The basic treatment protocol for patients with CL/P consists of pre-surgical orthopedic appliances, surgical repair of the lip and palate, and treatment of associated complications such as speech, otology, and dental anomalies. Pre-surgical orthopedics have many important advantages which include: reducing the gap between the alveolar and maxillary segments, aligning and correcting the nasal cartilage, lengthening the columella, minimizing the formation of scar tissue after surgery due to the cleft segments being closer to each other, reducing the need for secondary surgery, enabling the surgeon to perform gingival perioplasty and ultimately preventing the need for secondary alveolar grafting, and producing more consistent postoperative results (6-9).

Nasoalveolar molding (NAM) is one of the important pre-surgical orthopedic techniques $(10,11)$ (Figure 1). Matsuo et al. (12) introduced a pre-surgical orthopedic technique that first focused on molding the cartilage. Grayson and Maull (13) applied the theory that newborn cartilaginous tissues are softer and their plasticity higher due to the level of estrogen transferred from the mother to the nasal cartilages of newborns with $C L / P$, introducing the NAM appliance and its benefits $(3,8)$.

Pediatricians are physicians who are responsible for monitoring the overall health of newborns, infants, and children. The early diagnosis of various dental conditions is critical in the success of patient treatment. Pediatricians who encounter patients with CL/P play an important role in determining and forming the appropriate multidisciplinary team care $(2,14)$. It is important to direct the parents of newborns with CL/P to a specialist as soon as possible for the purpose of applying a NAM appliance. This responsibility falls to pediatricians, who are usually the first clinicians to encounter newborns with $\mathrm{CL} / \mathrm{P}$.

The purpose of this study was to determine the level of knowledge and awareness of pediatricians regarding NAM and the pre-surgical orthopedic treatment of newborns with $\mathrm{CL} / \mathrm{P}$.

\section{Materials and Methods}

We performed a questionnaire study on pediatricians who were randomly selected from all over Türkiye. The study was approved by the Clinical Research Ethics Committee of Ordu University (no: 2021/9). The survey was specifically designed by the researchers using Google Forms ( $)$. Emails were sent to 600 pediatricians. Of the 600 pediatricians, 112 agreed to participate in the study. The sample size was calculated in the G*Power (version 3.1.9.2; Axel Buchner, Universität Düsseldorf, Düsseldorf, Germany) program and the total sample size required to detect a medium-sized effect with $90 \%$ power (effect size: 0.60) was found to

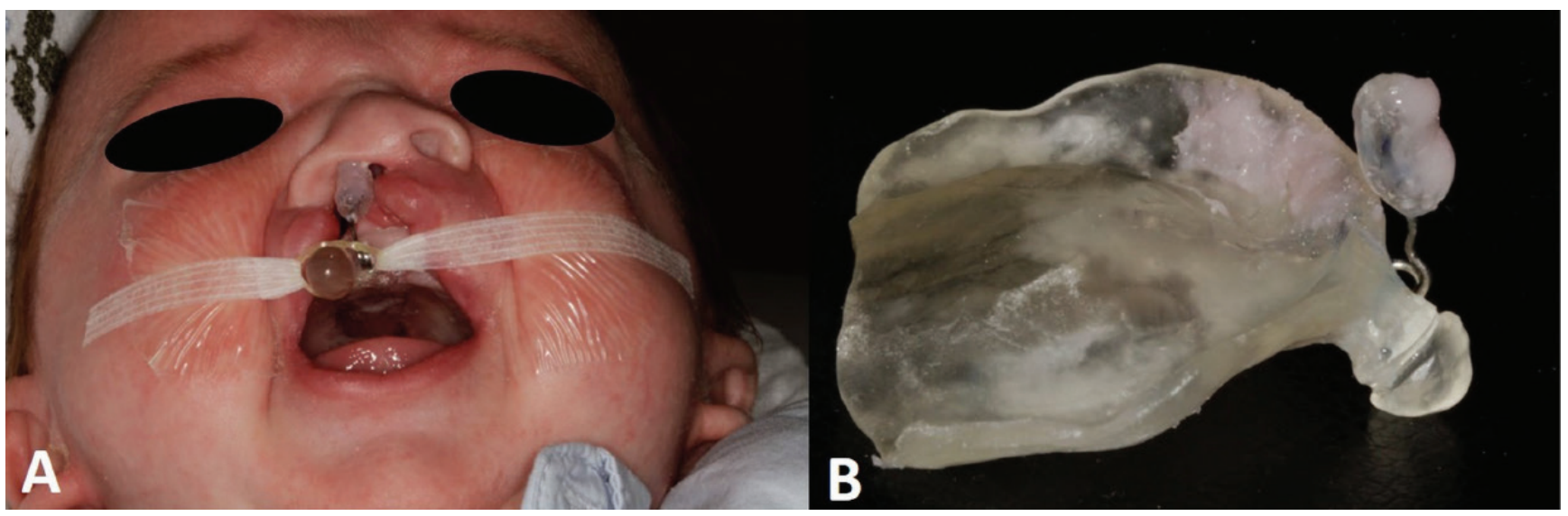

Figure 1. Nasoalveolar molding appliance 
be 98 for this study. The questionnaire consisted of 10 questions in 2 parts. First, demographic information such as title, workplace, and years of experience was recorded. Pediatricians who had greater than 5 years' experience in pediatrics were described as experienced, while those who had 5 or less than 5 years of pediatric experience were described as inexperienced. In the second part, participants were queried about their knowledge of $\mathrm{CL} / \mathrm{P}$ and the NAM appliance.

\section{Statistical Analysis}

Statistical analysis was performed using the SPSS software (SPSS for Windows version 20.0; SPSS Inc, Chicago, Illinois). Descriptive statistics were determined for the evaluated parameters. For categorical variables, percentages were calculated. Pearson chi-square and Fisher's exact tests were used to compare the pediatricians' level of knowledge about pre-surgical orthopedic treatment and the NAM appliance. Logistic regression analyses were performed to evaluate the effect of gender and years in practice on knowledge and referral to specialists for presurgical orthopedics and NAM therapy. Gender, years of experience, number of cases seen, and workplace served as the independent variables, while knowledge and referral to specialists were the dependent variables. Model fit was checked using the Hosmer-Lemeshow test. Statistical significance was accepted at $p<0.05$.

\section{Results}

A total of 112 pediatricians responded to the questionnaire, with a higher number of females (72.3\%) than males (27.7\%). Of the participants, 50\% reported working as pediatricians for less than 5 years, and $50 \%$ reported working for more than 5 years. Most of the respondents reported working in a university hospital (50\%), while $32.1 \%$ reported working in a state hospital and $17.9 \%$ reported working in a private hospital/practice. The demographic characteristics of pediatricians as well as their knowledge of NAM can be seen in Table I. There was a statistically significant number of pediatricians who had encountered newborns with $\mathrm{CL} / \mathrm{P}$ and had knowledge of NAM therapy $(p<0.001)$.

In the questionnaire, $84 \%$ of pediatricians reported that they redirect newborns with $\mathrm{CL} / \mathrm{P}$ to plastic and reconstructive plastic surgeons, $52 \%$ redirect to an orthodontist, and $39 \%$ redirect to a dentist. All respondents reported referring newborns with $\mathrm{CL} / \mathrm{P}$ to at least one of these three departments (Figure 2).

There was a statistically significant difference in the knowledge level regarding the preoperative treatment of newborns with $\mathrm{CL} / \mathrm{P}$ between experienced and inexperienced pediatricians ( $p>0.05)$. Moreover, there was a statistically significant difference between experienced and inexperienced pediatricians in the number of newborns with $C L / P$ examined $(p<0.05)$. The difference in knowledge level regarding NAM treatment and applications for newborns with $\mathrm{CL} / \mathrm{P}$ between experienced and inexperienced pediatricians was not statistically significant $(p>0.05)$ (Table II).

According to the results of multivariate analysis for the effect of gender, workplace, experience, and number of

Table I. Demographic characteristics of pediatricians about the knowing NAM therapy

\begin{tabular}{|c|c|c|c|c|c|}
\hline & Parameter & $\%$ & Yes & No & p-value \\
\hline \multirow{2}{*}{ Gender } & Male & 27.7 & 12 & 19 & \multirow{2}{*}{$0.775^{\alpha}$} \\
\hline & Female & 72.3 & 29 & 52 & \\
\hline \multirow{4}{*}{ Number of cases } & None & 8.9 & 1 & 9 & \multirow{4}{*}{$0.001^{\beta}$} \\
\hline & $0-10$ & 68.8 & 23 & 54 & \\
\hline & $11-20$ & 12.5 & 9 & 5 & \\
\hline & $>20$ & 9.8 & 8 & 3 & \\
\hline \multirow{2}{*}{ Experience } & 5 or less than 5 years & 50 & 17 & 39 & \multirow{2}{*}{$0.170^{\alpha}$} \\
\hline & More than 5 years & 50 & 24 & 32 & \\
\hline \multirow{3}{*}{ Workplace } & University hospital & 50 & 23 & 33 & \multirow{3}{*}{$0.409^{\alpha}$} \\
\hline & State hospital & 32.1 & 10 & 26 & \\
\hline & Private practice & 17.9 & 8 & 12 & \\
\hline
\end{tabular}


cases encountered on knowledge of NAM therapy, a linear regression was only seen between the number of cases encountered and years of experience (Table III). There was no correlation between years of experience and the level of knowledge about the NAM appliance.

\section{Discussion}

$\mathrm{CL} / \mathrm{P}$ is one of the most common orofacial malformations, with orofacial clefts occurring in approximately 1 in 700 live births $(15,16)$. Pediatricians play an important role in forming the multidisciplinary treatment team for patients with $\mathrm{CL} / \mathrm{P}$. Our study aimed to measure the level of pediatrician awareness regarding NAM therapy, which is one of the most prevalent pre-surgical orthopedic treatments for $\mathrm{CL} / \mathrm{P}$.

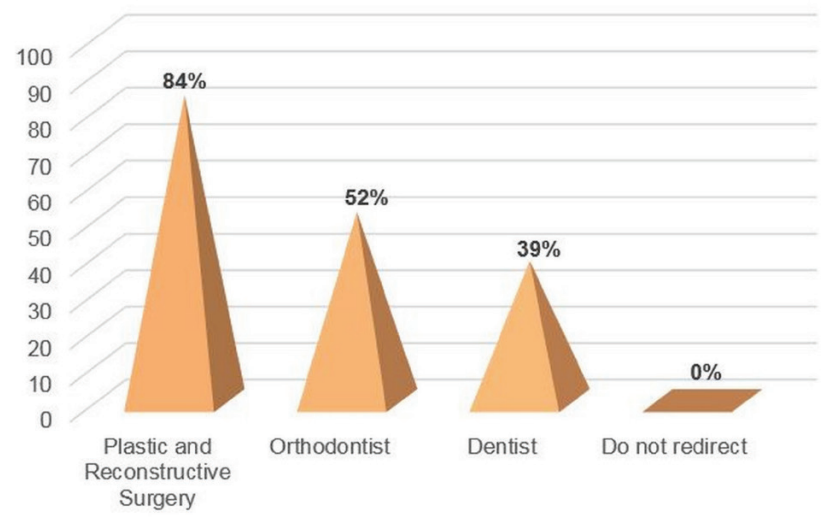

Figure 2. The $\%$ distributions of the pediatricians redirect $C L / P$ newborns related departments for NAM

NAM: Nasoalveolar molding
Orofacial clefts are one of the most common congenital anomalies, with a higher prevalence than neural tube defects, though the prevalence of cardiovascular malformations is higher $(17,18)$. In the present study, 10 pediatricians $(9 \%)$ reported having never been at the birth of a newborn with $\mathrm{CL} / \mathrm{P}$, while 11 pediatricians reported taking part in the birth of more than 20 newborns with $\mathrm{CL} / \mathrm{P}$. Experienced pediatricians were found to have seen more cases of $\mathrm{CL} / \mathrm{P}$ than inexperienced pediatricians, and this difference was statistically significant.

In the 1950s, modern methods of pre-surgical orthopedics began with the acrylic plates used by McNeil (19) in patients with CL/P. McNeil (19) used these plates to reduce the space between the hard palate and the alveolar segments. Many modifications of this appliance have since been used for orthopedic treatment before surgery, being used either actively or passively depending on the indication for its use. Grayson and Maull (13) introduced the NAM appliance that was based on the shaping of the nasoalveolar segments in the first weeks after a newborn's birth, and this is now one of the most up-to-date appliances used for pre-surgical orthopedic treatment. The NAM appliance is one of the latest and most important treatment techniques as it enables the surgeon and patient to benefit from a cleft deformity repair that is of minimal severity $(3,7,13)$. While approximately $67 \%$ of the pediatricians who participated in the survey stated that they had knowledge of pre-surgical orthopedic treatments, the remaining participants stated that they did not have any knowledge on this subject. Of the pediatricians

Table II. Responses of pediatricians according to their level of knowledge about preoperative treatment of CL/P

\begin{tabular}{|c|c|c|c|c|}
\hline & Answer & $\begin{array}{l}\text { Experience less } \\
\text { than } 5 \text { years }\end{array}$ & $\begin{array}{l}\text { Experience more } \\
\text { than } 5 \text { years }\end{array}$ & p-value \\
\hline \multirow{2}{*}{$\begin{array}{l}\text { Do you have any information about presurgical orthopedics performed } \\
\text { before surgical treatment in } \mathrm{CL} / \mathrm{P} \text { newborns? }\end{array}$} & Yes & 33 & 42 & \multirow{2}{*}{$0.071^{\alpha}$} \\
\hline & No & 23 & 14 & \\
\hline \multirow{4}{*}{ How many $\mathrm{CL} / \mathrm{P}$ newborn patients have you examined? } & None & 7 & 3 & \multirow{4}{*}{$0.021^{\beta}$} \\
\hline & $0-10$ & 43 & 34 & \\
\hline & $11-20$ & 4 & 10 & \\
\hline & $>20$ & 2 & 9 & \\
\hline \multirow{2}{*}{ Have you heard about the use NAM therapy in newborns with CL/P? } & Yes & 38 & 43 & \multirow{2}{*}{$0.291^{\alpha}$} \\
\hline & No & 15 & 13 & \\
\hline \multirow{2}{*}{ Do you know what NAM and its' purpose? } & Yes & 17 & 24 & \multirow{2}{*}{$0.170^{\alpha}$} \\
\hline & No & 39 & 32 & \\
\hline \multirow{2}{*}{ Do you know who performed the NAM therapy? } & Yes & 18 & 27 & \multirow{2}{*}{$0.083^{\alpha}$} \\
\hline & No & 38 & 29 & \\
\hline
\end{tabular}


without knowledge of pre-surgical orthopedic treatment, $62 \%$ consisted of pediatricians with less than 5 years of experience.

When asked about NAM and its purpose in patients with $\mathrm{CL} / \mathrm{P}, 41$ providers (37\%) stated that they had knowledge of this appliance and its purpose, while 71 providers (63\%) stated that they did not have any knowledge of NAM. There was no statistical difference between the experienced and inexperienced groups regarding this question. According to this result, pediatricians in Turkey do not have enough knowledge of NAM therapy and its purpose. Such a low rate of awareness is surprising, as the NAM appliance is used quite frequently in pre-surgical orthopedic treatment for patients with $\mathrm{CL} / \mathrm{P}$. In a previous study that aimed to measure the attitude of cleft care specialists working in Africa towards the NAM appliance, NAM was only used by orthodontists and consequently, orthodontists had a stronger positive attitude towards pre-surgical orthopedics than other specialists (general surgeons, plastic surgeons, oral and maxillofacial surgeons, otolaryngologists, anesthetists, and general nurses). Similar to the results of our study, the popularity of NAM was limited among pediatricians (20).

We could not identify any other survey study assessing pediatrician awareness and knowledge of pre-surgical orthopedic treatment and the NAM appliance in the literature, which limited the comparisons with our study findings. When asked if providers knew who performed NAM applications, 45 providers (40\%) stated that they knew, while 67 providers (60\%) stated that they did not know. This was a similar response to the response we received to the previous question.

Another question asked to pediatricians in the questionnaire was, "Who do you refer the newborns with CL/P to?" All pediatricians reported that they direct patients to at least one specialist, $84 \%$ to plastic and reconstructive surgeons, 52\% to orthodontists, and 39\% to dentists. CL/P should be treated by a multidisciplinary team and this approach involves many specialists

Table III. Multivariate analysis for the effect gender, workplace, experience and number of cases on knowledge NAM therapy

\begin{tabular}{|c|c|c|c|c|c|}
\hline & Parameter & & p-value & OR & $95 \% \mathrm{Cl}$ \\
\hline \multirow{11}{*}{$\begin{array}{l}\text { Do you have any information about orthopedic } \\
\text { applications performed before surgical treatment of lip- } \\
\text { palate cleft in newborn with lip-palate cleft? }\end{array}$} & \multirow{2}{*}{ Gender } & $\mathrm{F}^{*}$ & \multirow{2}{*}{0.457} & \multirow{2}{*}{0.69} & \multirow{2}{*}{$0.26-1.82$} \\
\hline & & M & & & \\
\hline & \multirow{2}{*}{ Experience } & Less than 5 years & \multirow{2}{*}{0.293} & \multirow{2}{*}{1.57} & \multirow{2}{*}{$0.68-3.61$} \\
\hline & & More than 5 years* & & & \\
\hline & \multirow{4}{*}{ Number of cases } & None* & & & \\
\hline & & $0-10$ & 0.012 & 23.33 & $1.99-273.29$ \\
\hline & & $11-20$ & 0.245 & 3.51 & $0.42-29.17$ \\
\hline & & $>20$ & 0.416 & 2.73 & $0.24-30.66$ \\
\hline & \multirow{3}{*}{ Workplace } & University hospital* & & & \\
\hline & & State hospital & 0.457 & 1.60 & $0.46-5.53$ \\
\hline & & Private practice & 0.396 & 1.76 & $0.48-6.49$ \\
\hline \multirow{11}{*}{ Do you know what NAM and its' purpose? } & \multirow{2}{*}{ Gender } & $\mathrm{F}^{*}$ & \multirow{2}{*}{0.775} & \multirow{2}{*}{0.88} & \multirow{2}{*}{$0.38-2.07$} \\
\hline & & M & & & \\
\hline & \multirow{2}{*}{ Experience } & Less than 5 years & \multirow{2}{*}{0.171} & \multirow{2}{*}{1.72} & \multirow{2}{*}{$0.79-3.74$} \\
\hline & & More than 5 years* & & & \\
\hline & \multirow{4}{*}{ Number of cases } & None* & & & \\
\hline & & $0-10$ & 0.011 & 24.00 & $2.06-279.62$ \\
\hline & & $11-20$ & 0.011 & 6.26 & $1.52-25.74$ \\
\hline & & $>20$ & 0.654 & 1.48 & $0.27-8.27$ \\
\hline & \multirow{3}{*}{ Workplace } & University hospital* & & & \\
\hline & & State hospital & 0.933 & 0.96 & $0.34-2.71$ \\
\hline & & Private practice & 0.350 & 1.73 & $0.55-5.50$ \\
\hline
\end{tabular}


including obstetrician-gynecologists, pediatricians, speech therapists, plastic surgeons, orthodontists, and dentists. Pediatricians, along with obstetriciangynecologists, are usually the first step in the treatment chain. Pediatricians' increased knowledge about presurgical treatment options affects the success of the treatment. Pre-surgical orthopedic applications are recommended to begin within the first few days after birth because the plasticity of the tissues is at its highest. The authors reported that the cartilaginous tissues of a newborn are softer, and their plasticity is higher due to the level of estrogen transferred from the mother $(3,8)$. Therefore, pediatricians should direct the family to the right specialist during this period.

Patients with $\mathrm{CL} / \mathrm{P}$ should be treated with a multidisciplinary approach that involves many specialists, with pediatricians being one of the most important. Narrowing of the cleft region and an unchanged sagittal dimension, reduction in the number of soft tissue revision and alveolar grafting surgeries, and achievement of excellent facial esthetics with minimal scar tissue are some of the main benefits of this therapy $(3,8,21)$.

\section{Study Limitations}

The present study has some limitations. This study was performed on pediatricians in a single country. It is important to obtain more global results can be achieved with larger sample numbers, including pediatricians from different countries.

\section{Conclusion}

It is critical to begin the pre-surgical orthopedic treatment process as soon as possible after birth to enhance treatment success. The results of our study indicate that pediatricians do not have sufficient knowledge of NAM and its purpose. Consequently, it is important to increase pediatrician awareness and knowledge regarding treatment alternatives for patients with $\mathrm{CL} / \mathrm{P}$, including NAM and presurgical orthopedic treatment.

\section{Ethics}

Ethics Committee Approval: The study was approved by the Ordu University Clinical Research Ethics Committee with approval number: 2021/09 and date: 07.01.2021.

Informed Consent: For this type of this study, the informed consent is not required.

Peer-review: Externally peer-reviewed.

\section{Authorship Contributions}

Concept: K.K.D., S.K.B., T.A., Data Collection or Processing: T.A., Analysis or Interpretation: S.K.B., Writing: K.K.D.

Conflict of Interest: The authors declared no conflict of interest.

Financial Disclosure: The authors declared that this study received no financial support.

\section{References}

1. Imani MM, Golchin N, Safaei M, et al. Methylenetetrahydrofolate reductase $\mathrm{C677T}$ polymorphism is not associated with the risk of nonsyndromic cleft lip/palate: An updated meta-analysis. Sci Rep 2020; 10:1531.

2. Lewis CW, Jacob LS, Lehmann CU; SECTION ON ORAL HEALTH. The primary care pediatrician and the care of children with cleft lip and/or cleft palate. Pediatrics 2017; 139:e20170628.

3. Altuğ AT. Presurgical Nasoalveolar Molding of Bilateral Cleft Lip and Palate Infants: An Orthodontist's Point of View. Turk J Orthod 2017; 30:118-25

4. Ji Y, Garland MA, Sun B, et al. Cellular and developmental basis of orofacial clefts. Birth Defects Res 2020; 112:1558-87.

5. Garland MA, Reynolds K, Zhou C). Environmental mechanisms of orofacial clefts. Birth Defects Res 2020; 112:1660-98.

6. Nakajima T, Yoshimura Y, Sakakibara A. Augmentation of the nostril splint for retaining the corrected contour of the cleft lip nose. Plast Reconstr Surg 1990; 85:182-6.

7. Maull D), Grayson BH, Brecht L, Cutting C. Long term effects of nasoalveolar molding. annual meeting of the American Cleft Palate Craniofacial Association. 1999. https://pubmed.ncbi. nlm.nih.gov/10499400/

8. Ross RB, MacNamera MC. Effect of presurgical infant orthopedics on facial esthetics in complete bilateral cleft lip and palate. Cleft Palate Craniofac ) 1994; 31:68-73.

9. Hotz M, Gnoinski W. Comprehensive care of cleft lip and palate children at Zürich University: a preliminary report. Am J Orthod 1976; 70:481-504

10. Yarholar LM, Shen C, Wangsrimongkol B, et al. The Nasoalveolar Molding Cleft Protocol: Long-Term Treatment Outcomes from Birth to Facial Maturity. Plast Reconstr Surg 2021; 147:787-94

11. Maliha SG, Kantar RS, Gonchar MN, et al. The Effects of Nasoalveolar Molding on Nasal Proportions at the Time of Nasal Maturity. Cleft Palate Craniofac ) 2021; 58:284-9.

12. Matsuo K, Hirose T, Tomono T, et al. Nonsurgical correction of congenital auricular deformities in the early neonate: a preliminary report. Plast Reconstr Surg 1984; 73:38-50.

13. Grayson BH, Maull D. Nasoalveolar molding for infants born with clefts of the lip, alveolus and palate. Cleft Lip and Palate, Springer, 2006. p. 451-8.

14. Ovsenik M, Primožič ). How to push the limits in the transverse dimension? Facial asymmetry, palatal volume and tongue posture in children with unilateral posterior cross bite: a threedimensional evaluation of early treatment. Orthod Fr 2014. 85:139-49. 
15. Cobourne MT. The complex genetics of cleft lip and palate. Eur J Orthod 2004; 26:7-16.

16. Yılmaz HN, Özbilen EÖ, Üstün T. The Prevalence of Cleft Lip and Palate Patients: A Single-Center Experience for 17 Years. Turk J Orthod 2019; 32:139-44.

17. Impellizzeri A, Giannantoni I, Polimeni A, Barbato E, Galluccio G. Epidemiological characteristic of Orofacial clefts and its associated congenital anomalies: retrospective study. BMC Oral Health 2019; 19:290.

18. Bianchi F, Calzolari E, Ciulli L, et al. Environment and genetics in the etiology of cleft lip and cleft palate with reference to the role of folic acid. Epidemiol Prev 2000; 24:21-7.
19. McNeil C. Orthodontic procedures in the treatment of congenital cleft palate. Dent Rec (London) 1950; 70:126-32.

20. Adeyemi AT, Bankole OO. Attitude of cleft care specialists in Africa towards pre-surgical orthopaedics. East Afr Med I 2012; 89:414-20.

21. Santiago PE, Grayson $B H$, Cutting $C B$, Gianoutsos MP, Brecht $L E$, Kwon SM. Reduced need for alveolar bone grafting by presurgical orthopedics and primary gingivoperiosteoplasty. Cleft Palate Craniofac ) 1998; 35:77-80. 